\title{
The Mechanisms and Molecular Targets of Compound Danshen dropping pill for heart disease caused by high altitude Based on Network Pharmacology and molecular docking
}

Jiayi Yan ${ }^{1,2 \#}$, Panpan Ruan ${ }^{2,3 \#}$, Yunxuan Ge ${ }^{2,4}$, Jing Gao ${ }^{2,5}$, Hongling Tan ${ }^{2}$, Chengrong Xiao ${ }^{2}$, Quansheng $\mathrm{Gao}^{6}$, Zhuo Zhang ${ }^{2 *}$,Yue $\mathrm{Gao}^{1,2^{*}}$

1.School of Traditional Chinese Medicine, Guangdong Pharmaceutical University, Guangzhou, China

2. Department of Pharmaceutical Sciences, Beijing Institute of Radiation Medicine, Beijing, China

3.School of Life Science, Heibei University, Baoding, China

4. College of Life Science and Bioengineering, Beijing University of Technology, Beijing, 100124, China

5. School of Pharmacy, Henan University, Kaifeng, 475004, China 6.Institute of enviromental and operational medicine, Academy of military medical sciences, Academy of military sciences., Tianjin, China. \#These authors contributed equally to this work *Correspondence: gaoyue@bmi.ac.cn zhangzhuo000@yeah.net Abstract 
Compound Danshen dropping pill (CDDP), a famous Chinese medicine formula, has been widely used to treat high altitude heart disease in China. However, its molecular mechanism, potential targets and bioactive ingredients remain elusive. In this study, network pharmacology, molecular docking and validation experiments were combined to investigate the effective active ingredients and molecular mechanisms of CDDP in the treatment of high altitude heart disease. Tan IIA may be a main active component of CDDP in the treatment of High-altitude heart disease via HIF-1/PI3K/Akt pathways.

Supplementary Table 1 : active components and corresponding targets

\begin{tabular}{lll}
\hline Node1 & Node2 & MolName \\
\hline MOL000006 & VEGFA & luteolin \\
MOL000098 & VEGFA & quercetin \\
MOL000098 & THBD & quercetin \\
MOL000098 & SOD1 & quercetin \\
MOL001601 & SLC6A4 & 1,2,5,6-tetrahydrotanshinone \\
MOL007041 & SLC6A4 & 2-isopropyl-8-methylphenanthrene-3,4-dione \\
MOL007049 & SLC6A4 & 4-methylenemiltirone \\
MOL007061 & SLC6A4 & Methylenetanshinquinone \\
MOL007094 & SLC6A4 & danshenspiroketallactone \\
MOL007100 & SLC6A4 & dihydrotanshinlactone \\
MOL007105 & SLC6A4 & epidanshenspiroketallactone \\
MOL007124 & SLC6A4 & neocryptotanshinone ii \\
MOL007145 & SLC6A4 & salviolone \\
MOL000358 & SLC6A4 & beta-sitosterol \\
MOL000098 & SERPINE1 quercetin \\
MOL001601 & SCN5A & 1,2,5,6-tetrahydrotanshinone \\
MOL002651 & SCN5A & Dehydrotanshinone II A \\
MOL007036 & SCN5A & 5,6-dihydroxy-7-isopropyl-1,1-dimethyl-2,3-dihydrophenanthren-4-one \\
MOL007041 & SCN5A & 2-isopropyl-8-methylphenanthrene-3,4-dione \\
MOL007045 & SCN5A & 3-hydroxytanshinone \\
MOL007069 & SCN5A & przewaquinone c \\
MOL007049 & SCN5A & 4-methylenemiltirone \\
MOL007061 & SCN5A & Methylenetanshinquinone \\
\hline
\end{tabular}




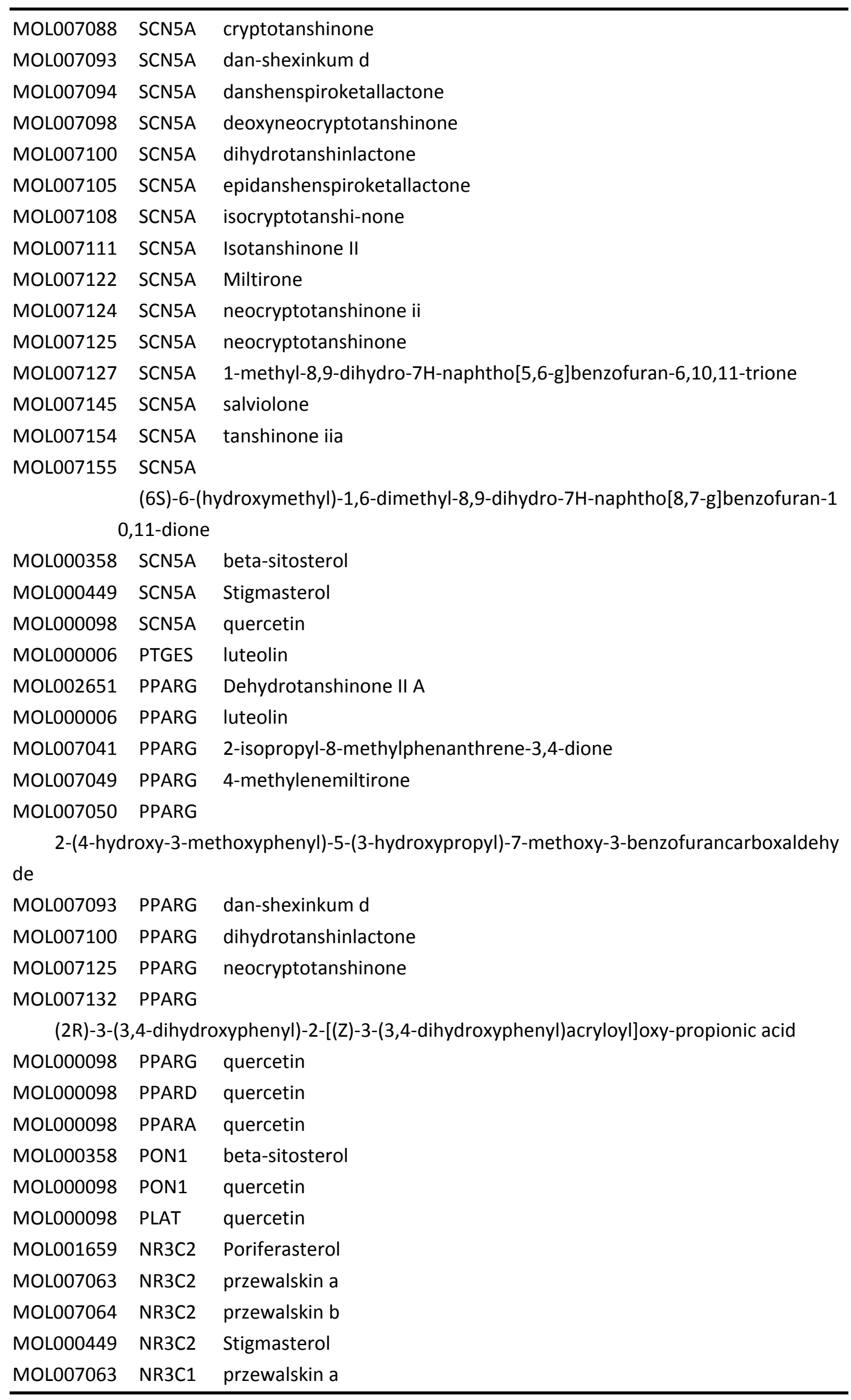




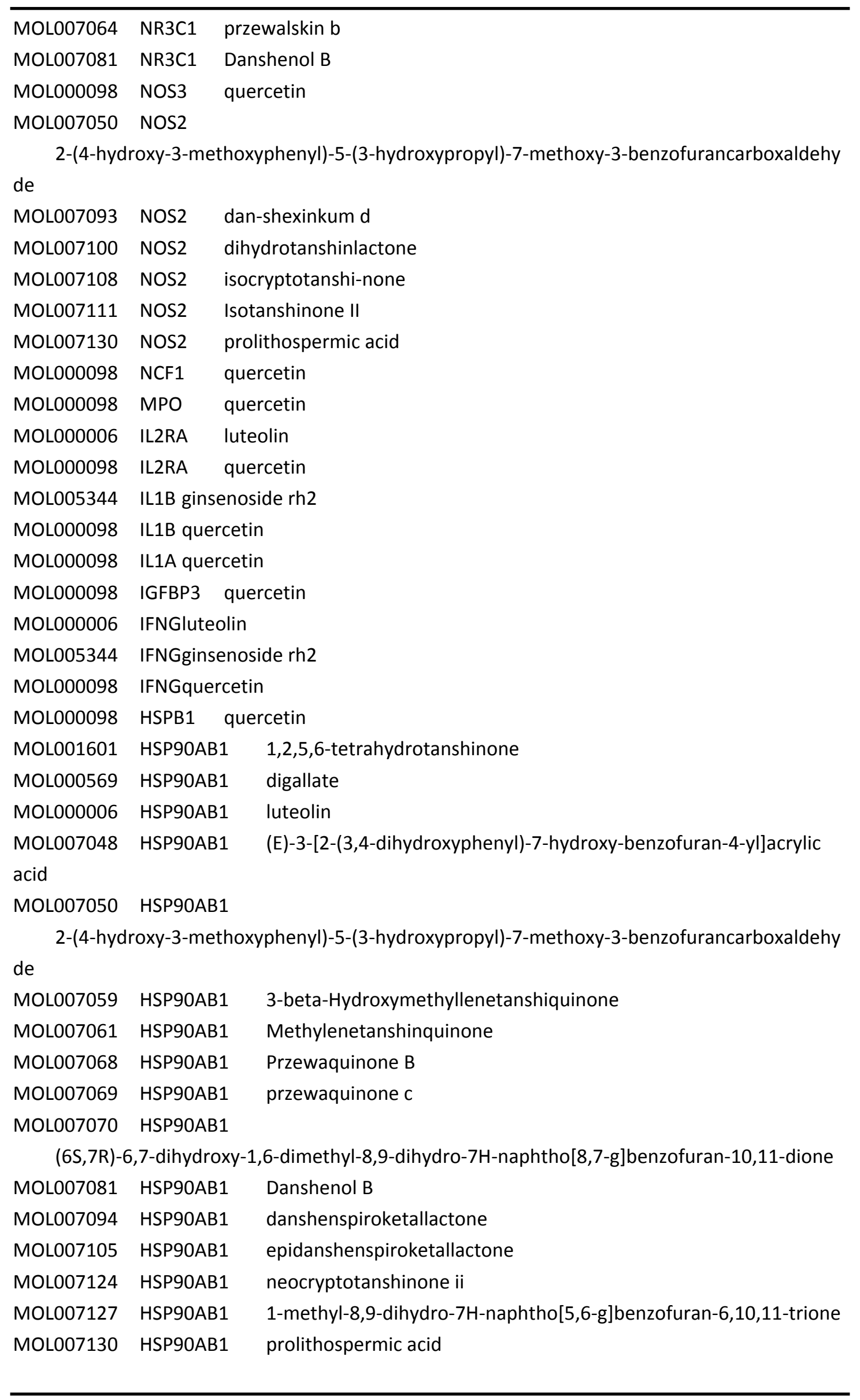


MOL007150 HSP90AB1

(6S)-6-hydroxy-1-methyl-6-methylol-8,9-dihydro-7H-naphtho[8,7-g]benzofuran-10,11-quino ne

MOL007151 HSP9OAB1 Tanshindiol B

MOL007152 HSP90AB1 Przewaquinone E

MOL000358 HSP90AB1 beta-sitosterol

MOL000098 HSP90AB1 quercetin

MOL000006 HMOX1 luteolin

MOL000098 HMOX1 quercetin

MOL000098 HIF1A quercetin

MOL000006 GSTP1 luteolin

MOL000098 GSTP1 quercetin

MOL000098 GSTM1 quercetin

MOL000098 GJA1 quercetin

MOL000098 F3 quercetin

MOL000006 EGFR luteolin

MOL000098 EGFR quercetin

MOL007154 EDNRA tanshinone iia

MOL007088 EDN1 cryptotanshinone

MOL007154 EDN1 tanshinone iia

MOL007154 ECE1 tanshinone iia

MOL000098 DIO1 quercetin

MOL007154 CYP3A4 tanshinone iia

MOL000098 CYP3A4 quercetin

MOL000098 CXCL8 quercetin

MOL000098 CAV1 quercetin

MOL000006 CASP9 luteolin

MOL000358 CASP9 beta-sitosterol

MOL000098 CASP9 quercetin

MOL000358 CASP8 beta-sitosterol

MOL000098 CASP8 quercetin

MOL001601 CA2 1,2,5,6-tetrahydrotanshinone

MOL007036 CA2 5,6-dihydroxy-7-isopropyl-1,1-dimethyl-2,3-dihydrophenanthren-4-one

MOL007049 CA2 4-methylenemiltirone

MOL007059 CA2 3-beta-Hydroxymethyllenetanshiquinone

MOL007061 CA2 Methylenetanshinquinone

MOL007069 CA2 przewaquinone $\mathrm{c}$

MOL007070 CA2

(6S,7R)-6,7-dihydroxy-1,6-dimethyl-8,9-dihydro-7H-naphtho[8,7-g]benzofuran-10,11-dione

MOL007081 CA2 Danshenol B

MOL007088 CA2 cryptotanshinone

MOL007093 CA2 dan-shexinkum d

MOL007094 CA2 danshenspiroketallactone

MOL007098 CA2 deoxyneocryptotanshinone 
MOL007100 CA2 dihydrotanshinlactone

MOL007108 CA2 isocryptotanshi-none

MOL007122 CA2 Miltirone

MOL007124 CA2 neocryptotanshinone ii

MOL007125 CA2 neocryptotanshinone

MOL007127 CA2 1-methyl-8,9-dihydro-7H-naphtho[5,6-g]benzofuran-6,10,11-trione

MOL007150 CA2

(6S)-6-hydroxy-1-methyl-6-methylol-8,9-dihydro-7H-naphtho[8,7-g]benzofuran-10,11-quino ne

MOL007151 CA2 Tanshindiol B

MOL007152 CA2 Przewaquinone E

MOL007154 BCL2 tanshinone iia

MOL000358 BCL2 beta-sitosterol

MOL000098 BCL2 quercetin

MOL002651 AR Dehydrotanshinone II A

MOL000006 AR luteolin

MOL007041 AR 2-isopropyl-8-methylphenanthrene-3,4-dione

MOL007049 AR 4-methylenemiltirone

MOL007050 AR

2-(4-hydroxy-3-methoxyphenyl)-5-(3-hydroxypropyl)-7-methoxy-3-benzofurancarboxaldehy de

MOL007058 AR formyltanshinone

MOL007085 AR Salvilenone

MOL007093 AR dan-shexinkum d

MOL007098 AR deoxyneocryptotanshinone

MOL007100 AR dihydrotanshinlactone

MOL007108 AR isocryptotanshi-none

MOL007111 AR Isotanshinone II

MOL007122 AR Miltirone

MOL007124 AR neocryptotanshinone ii

MOL007130 AR prolithospermic acid

MOL007132 AR

(2R)-3-(3,4-dihydroxyphenyl)-2-[(Z)-3-(3,4-dihydroxyphenyl)acryloyl]oxy-propionic acid

MOL000098 AR quercetin

MOL000006 AKT1 luteolin

MOL000098 AKT1 quercetin

MOL001601 ADRB2 1,2,5,6-tetrahydrotanshinone

MOL002651 ADRB2 Dehydrotanshinone II A

MOL007036 ADRB2 5,6-dihydroxy-7-isopropyl-1,1-dimethyl-2,3-dihydrophenanthren-4-one

MOL007041 ADRB2 2-isopropyl-8-methylphenanthrene-3,4-dione

MOL007045 ADRB2 3-hydroxytanshinone

MOL007049 ADRB2 4-methylenemiltirone

MOL007059 ADRB2 3-beta-Hydroxymethyllenetanshiquinone

MOL007061 ADRB2 Methylenetanshinquinone 


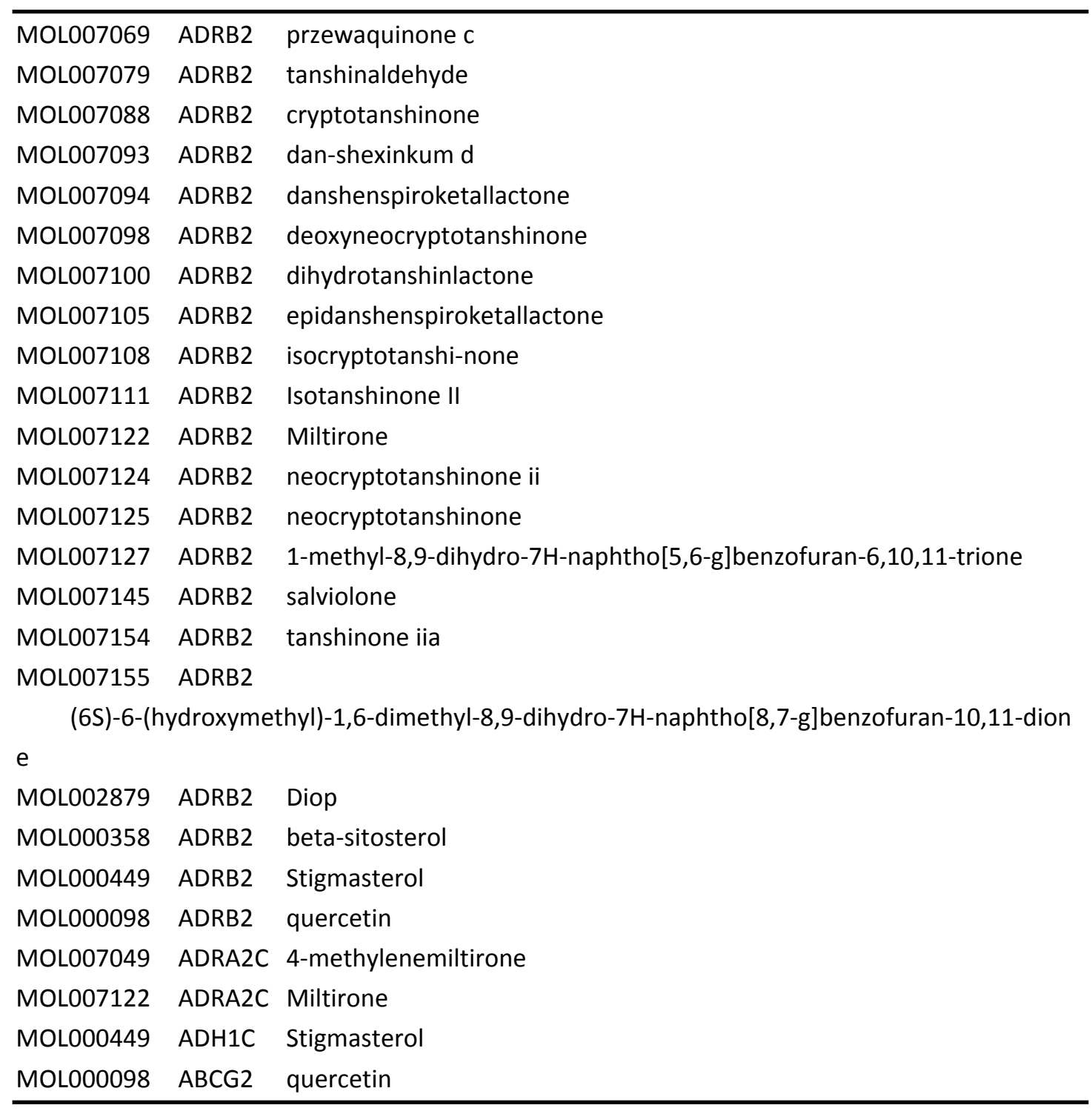

